\title{
Simulation study for the Electromagnetic Calorimeter Trigger system at the Belle II Experiment
}

\section{I.S.Lee*}

Dept. of Physics, Hanyang University E-mail: is lee@hep. hanyang.ac.kr

\section{S.H.Kim}

Dept. of Physics, Hanyang University

E-mail: sunghyun.kimebelle2.org

\section{C.H.Kim}

Dept. of Physics, Hanyang University

E-mail: chkim@hep.hanyang.ac.kr

\section{H.E.Cho}

Dept. of Physics, Hanyang University

E-mail: hecho@hep. hanyang.ac.kr

\section{Y.J.Kim}

Dept. of Physics, Korea University

E-mail: rladudwns118@korea.ac.kr

\section{Y.Unno}

Dept. of Physics, Hanyang University

E-mail: yunnolpost.kek.jp

\section{B.G.Cheon}

Dept. of Physics, Hanyang University

E-mail: bgcheon@hanyang.ac.kr

The Belle II experiment at KEK in Japan started beam collision from early of 2018 to probe a New Physics beyond the Standard Model by measuring CP violation phenomena and rare decays of beauty, charm quark and tau lepton. The experiment is performed at the SuperKEKB e+e- collider with $80 \times 10^{34} \mathrm{~cm}^{-2} \mathrm{~s}^{-1}$ as an ultimate instantaneous luminosity. As a severe beam background environment is highly anticipated, a detail simulation study of the Belle II calorimeter trigger system is very crucial to operate Belle II Trigger/DAQ system stably. We report simulation results on various trigger logic and efficiencies using physics and beam background events upon the Belle II Geant4-based analysis framework called Basf2.

ICHEP 2018, International Conference on High Energy Physics

4-11 July 2018

Seoul, Korea

${ }^{*}$ Speaker. 


\section{Introduction}

The Belle II experiment at KEK in Japan is ready for data-taking to probe New Physics beyond the Standard Model by measuring CP violation phenomena precisely and rare decays of beauty, charm quark and tau lepton. Because an anticipated beam background level is 50 times higher than the case of Belle in the target luminosity, $80 \times 10^{34} \mathrm{~cm}^{-2} \mathrm{~s}^{-1}$, the robust and flexible trigger system is indispensable to operate the Belle II detector smoothly. The basic framework and idea of Belle II electromagnetic calorimeter (ECL) trigger system are same as the case of the Belle[1, 2, 3]. The performance of trigger algorithms should be confirmed using ECL Trigger Simulation (TSim-ecl) package that is a $\mathrm{C}++$ based program implemented in the Belle II Geant4-based analysis framework called Basf2.

\section{Trigger logic study with TSim-ecl}

To determine the event-timing of the physics events, Belle experiment use the fastest timing from Trigger cell(TC) hits, where TC consists of $4 \times 4$ crystals of Electro-magnetic calorimeter(ECL). In Belle II, we determine the event-timing as the most energetic TC timing. The newly introduced method is around 2 times better resolution (3.7 ns) than the method in Belle (7.2 ns).

In e+e- collider, bhabha process is a main background having not only the highest event rate but similar event topology with low multiplicity process such as tau and Initial state radiation events. In order to suppress the bhabha events in previous Belle experiment, a 2-D $(r-\phi)$ back-to-back topology is required. This method has a weak point that misidentifies some of low multiplicity process as a bhabha event. In order to avoid such case, we use the 3-D $(r-\theta-\phi)$ back-to-back topology with tighter bhabha veto trigger than the Belle. To distinguish bhabha process, we find particle clusters. Then we distinguish the clusters making back-to-back topology through $\theta$ and $\phi$ direction. Finally, we apply energy threshold, $3 \mathrm{GeV}$ for each cluster and 4.5 $\mathrm{GeV}$ for one of cluster. Table 1 shows that 3-D logics gives better performance than 2-D logics.

Table 1: Bhabha trigger efficiency for Bhabha and low-multiplicity processes (\%)

\begin{tabular}{ccc}
\hline Sample & 2 -D & 3 -D \\
\hline \hline Bhabha & 91.8 & 91.8 \\
\hline $\operatorname{ISR}\left(e^{+} e^{-} \rightarrow \gamma \pi^{+} \pi^{-}\right)$ & 0.4 & 0.1 \\
\hline$\tau \rightarrow e \gamma$ & 3.7 & 0.4 \\
\hline$\tau \rightarrow \mu \gamma$ & 2.0 & 0.1 \\
\hline \hline
\end{tabular}

\section{References}

[1] A. Abashian et al. (Belle Collaboration), The Belle detector, Nucl. Instr. Meth. A 479 (2002) 117.

[2] Y. Iwasaki et al., Level 1 Trigger System for the Belle II experiment, IEEE Trans. Nucl. Sci, 58 (2011) 1807-1815.

[3] S.H. Kim, I.S. Lee, Y. Unno and B.G. Cheon,Status of the Electromagnetic Calorimeter Trigger system at the Belle II experiment ,JINST 12 C09004 (2017) 